\title{
Subcellular Localization of Maspin Correlates with Histone Deacetylase 1 Expression in Human Breast Cancer
}

\author{
MAKOTO WAKAHARA ${ }^{1,2}$, TOMOHIKO SAKABE ${ }^{1}$, YASUAKI KUBOUCHI ${ }^{1,2}$, \\ KEIKO HOSOYA ${ }^{2}$, YUMI HIROOKA ${ }^{2}$, YOHEI YURUGI ${ }^{1,2}$, KANAE NOSAKA $^{1}$, \\ TATSUSHI SHIOMI ${ }^{1}$, HIROSHIGE NAKAMURA ${ }^{2}$ and YOSHIHISA UMEKITA ${ }^{1}$ \\ ${ }^{1}$ Division of Organ Pathology, Department of Pathology, and ${ }^{2}$ Division of General Thoracic Surgery, \\ Department of Surgery, Faculty of Medicine, Tottori University, Tottori, Japan
}

\begin{abstract}
Background/Aim: Maspin is known to be a tumor suppressor protein. Its nuclear localization and endogenous inhibition of histone deacetylase 1 (HDAC1) are considered crucial for its tumor suppressor activity. However, it remains unclear whether subcellular localization of maspin correlates with HDAC1 expression level in human breast cancer. Patients and Methods: Immunohistochemical analyses were performed on 164 resected specimens of invasive breast carcinoma using antibodies for maspin and HDAC1. Subcellular localization of maspin protein and HDAC1 $\mathrm{mRNA}$ expression level in two human breast cancer cell lines (MCF7, MDA-MB-231) and mammary epithelial cell line (MCF10) were analyzed by immunofluorescence and quantitative polymerase chain reaction, respectively. Results: The frequency of cytoplasmiconly, pancellular (combined nuclear and cytoplasm) and no staining of maspin were $31 \%, 14.0 \%$ and $55 \%$, respectively. The cytoplasmic-only subgroup showed significantly higher histological grade $(p=0.004)$, negative progesterone receptor status $(p=0.003)$ and shorter disease-free survival compared to the pancellular subgroup ( $p=0.043)$. High HDACI expression was observed in $60 \%$ of cases and was significantly correlated with cytoplasmic-only staining compared to pancellular $(p<0.001)$ or no staining $(p=0.004)$. Immunofluorescence analysis revealed that maspin protein was localized mainly in the cytoplasm in MCF7 and MDAMB-231 cells, while in both the nucleus and cytoplasm in MCF10A cells. HDAC1 mRNA levels were significantly upregulated in MCF7 and MDA-MB-231 cells compared to MCF10A cells $(p<0.001)$. Conclusion: High HDAC1
\end{abstract}

Correspondence to: Prof. Yoshihisa Umekita, Division of Organ Pathology, Department of Pathology, Faculty of Medicine, Tottori University, 86 Nishicho, Yonago, Tottori 683-8503, Japan. Tel: +81 859386053, Fax +81 859386050, e-mail: yume@med.tottori-u.ac.jp

Key Words: Maspin, histone deacetylase 1, breast cancer, immunohistochemistry. expression may contribute to the aggressiveness of human breast cancer with cytoplasmic-only expression of maspin.

Maspin, a unique member of the serine protease inhibitor (serpin) superfamily, was identified originally as a tumor suppressor that is expressed in normal breast epithelial cells but is down-regulated in breast cancer cells (1). Maspin has been shown to inhibit both tumor growth and metastasis in animal models and cancer cell lines, and it has pro-apoptotic, antimetastatic and anti-angiogenic properties, exerting an inhibitory effect on cancer cell motility, invasiveness and metastatic ability (2). Although the exact biochemical pathways leading to these biological endpoints remain elusive, several studies have characterized the association between maspin expression and clinicopathological factors in breast cancer patients (3-6). We have also reported that cytoplasmic-only expression of maspin could be a poor prognostic indicator in breast cancer patients (7-9). On the other hand, it has been reported that the nuclear localization of maspin in cancer cells was necessary for its inhibition of tumor progression and that the endogenous inhibition of histone deacetylase 1 (HDAC1) could be one of the tumor suppressive mechanisms of maspin (10-12). Taken together, we hypothesized that cytoplasmic-only expression of maspin might be positively correlated with high expression of HDAC1 in human breast cancer. To our knowledge, no study has reported the association between subcellular localization of maspin protein and HDAC1 protein expression in human breast cancer tissue samples. The aim of the present study was to clarify whether subcellular localization of maspin correlates with HDAC1 expression level in human breast cancer in order to gain insight of the tumor suppressive mechanisms of maspin.

\section{Patients and Methods}

Patients and tumors specimens. From January 2008 to December 2011 at Tottori University Hospital, Japan, 227 consecutive female patients underwent a surgical resection of breast cancer diagnosed as invasive carcinoma of no special type according to the World 
Health Organization classification of breast cancer (13). Sixty-three cases were excluded due to a history of neoadjuvant treatment (38 cases), microinvasive carcinoma (9 cases), distant metastasis (5 cases), unavailable tumor specimen ( 5 cases), bilateral breast cancer (4 cases) and short follow-up period ( 2 cases). Thus, 164 patients were included in this analysis. The patients' clinicopathological data were obtained from their hospital medical records. The histopathological parameters including lymph node metastasis, estrogen receptor (ER), progesterone receptor (PR) and human epidermal growth factor receptor 2 (HER2) statuses were retrieved from the patients' pathology reports. Histological grades were determined according to the criteria of Elston and Ellis (14), and the grading was independently evaluated by M.W. and Y.U. Tumor stage was determined based on the seventh tumor-node-metastasis (TNM) classification of breast cancer (15). The study was approved by the ethics committee of the Faculty of Medicine, Tottori University (approval number: 1608A083; 15 August 2016).

Immunohistochemistry. All specimens were fixed in $10 \%$ neutrally buffered formalin and embedded in paraffin. The sections ( $4 \mu \mathrm{m}$-thick) were deparaffinized and endogenous peroxidase activity was blocked. The specimens were then pretreated in citrate buffer $(0.01 \mathrm{M}, \mathrm{pH} 6.0)$ in a microwave oven (IMB-T17-5/6; Irisohyama, Sendai, Japan) for 15 min at $700 \mathrm{~W}$ for maspin, or a water bath at $95^{\circ} \mathrm{C}$ for $10 \mathrm{~min}$ for HDAC1. After the pretreated specimens had cooled to room temperature, non-specific binding was blocked by blocking buffer (Block Ace; Megmilk Snow Brand, Sapporo, Japan). Next, the sections were incubated at $4^{\circ} \mathrm{C}$ overnight with a monoclonal antibody to maspin (clone EAW24, diluted 1:200; Leica Biosystems, Newcastle, UK) or at room temperature for $30 \mathrm{~min}$ with polyclonal antibody to HDAC1 (clone ab19845, diluted 1:3600; Abcam, Cambridge, MA, USA). The sections for maspin were incubated with biotinylated anti-mouse IgG antibody (BA-2000, diluted 1:150; Vector Laboratories, Burlingame, CA) for $20 \mathrm{~min}$, followed by streptavidin biotinylated horseradish peroxidase (HRP) conjugate (diluted 1:150; Invitrogen, Camarillo, CA, USA) for $20 \mathrm{~min}$. The sections for HDAC1 were incubated with the EnVision+ System HRP (Dako, Glostrup, Denmark). Finally, all of the sections were incubated with diaminobenzidine (DAB) solution (liquid $\mathrm{DAB}+$ substrate, imidazole-HCI buffer, $\mathrm{pH} 7.5$, containing hydrogen peroxide and an antimicrobial agent; Dako) for $4 \mathrm{~min}$, and counterstained with hematoxylin.

Evaluation of immunohistochemical findings. The cells were considered positive for maspin expression when strong cytoplasmiconly staining was identified. Strong staining was defined as the staining intensity equal to that of myoepithelial cells in normal breast tissue, and it served as an internal positive control if included. Tumors with $>10 \%$ positive cells were considered maspin-positive as described previously (8). The subcellular localization of maspin was classified into four categories; cytoplasmic-only, pancellular (combined nuclear and cytoplasmic), nuclear-only, and no staining. The evaluation of HDAC1 expression status was performed according to the methods described by Seo et al. (16). Briefly, the intensity $(0$, negative; 1 , weak; 2 , moderate; 3 , strong) and the proportion of positive cells $(0$, negative; $1,<10 \% ; 2,10-33 \% ; 3,33$ $66 \% ; 4, \geq 66 \%$ ) scores were calculated through multiplication and cases were divided into two groups according to their scores as follows: low expression (scores, 0-6) and high expression (scores, 8-12). All slides were evaluated by M.W. and Y.U. independently who were blinded to the patients' clinicopathological data.
Cell culture. The human mammary epithelial cell line MCF10A and the human breast cancer cell line MDA-MB-231 were purchased from the American Type Culture Collection (ATCC, Manassas, VA, USA). MCF10A cells were maintained in mammary epithelial cell growth medium (MEGM Bullet Kit; Lonza, Walkersville, MD, USA) supplemented with $100 \mathrm{ng} / \mathrm{mL}$ cholera toxin at $37^{\circ} \mathrm{C}$ with $5 \% \mathrm{CO}$. MDA-MB-231 cells were maintained in Leibovitz's L-15 Medium (Sigma-Aldrich, St. Louis, MO, USA) supplemented with $10 \%$ fetal bovine serum (FBS; Biological Industries Ltd. Kibbutz Beit Haemek, Israel) and L-glutamine at $37^{\circ} \mathrm{C}$ with $0 \% \mathrm{CO}_{2}$. The human breast cancer cell line MCF7 was purchased from the Riken BioResource Center Cell Bank (Riken BRC Cell Bank, Ibaraki, Japan) and cultured in Dulbecco's Modified Eagle Medium (DMEM; Nissui Pharmaceutical, Tokyo, Japan) supplemented with 10\% FBS, glucose, and L-glutamine at $37^{\circ} \mathrm{C}$ with $5 \% \mathrm{CO}_{2}$.

Immunofluorescence analysis. MCF10A $\left(4.0 \times 10^{4}\right.$ cells $\left./ \mathrm{cm}^{2}\right), \mathrm{MCF} 7$ $\left(2.0 \times 10^{4}\right.$ cells $\left./ \mathrm{cm}^{2}\right)$, and MDA-MB-231 $\left(4.0 \times 10^{4}\right.$ cells $\left./ \mathrm{cm}^{2}\right)$ cells were separately seeded in 8 -well chamber slides (Nalgen Nunc International, Rochester, NY, USA) and incubated for $24 \mathrm{~h}$. After incubation, the cells were fixed with $4 \%$ paraformaldehyde at room temperature for $15 \mathrm{~min}$ and permeabilized with ice-cold $100 \%$ methanol at $-20^{\circ} \mathrm{C}$ for $10 \mathrm{~min}$. These samples were blocked in $10 \%$ goat serum/ phosphate-buffered saline (PBS) at room temperature for $60 \mathrm{~min}$ and subsequently incubated with the anti-Maspin primary antibody (G167-70, diluted 1:10; BD Pharmingen, Franklin Lakes, NJ, USA) at $4^{\circ} \mathrm{C}$ overnight, followed by incubation with Alexa Fluor conjugated anti-mouse secondary antibody (Molecular Probes, Leiden, Netherlands). Nuclear counterstaining was performed using DAPI (Molecular Probes, Leiden, Netherlands). Immunofluorescence images were detected using an FV1000 confocal microscope (Olympus, Tokyo, Japan).

RNA extraction and quantitative polymerase chain reaction. Total RNA from MCF10A, MCF7, and MDA-MB-231 cells was extracted using TRIzol Reagent (Thermo Fisher Scientific, Waltham, MA, USA) and reverse-transcribed into complementary DNA (cDNA) using the High-Capacity RNA-to-cDNA Kit (Thermo Fisher Scientific, Waltham, MA, USA) according to manufacturer's protocol. We measured the gene expression levels by performing TaqMan Gene Expression Assays (Thermo Fisher Scientific, Waltham, MA, USA) with the following gene-specific primers: GAPDH, Human GAPDH Endogenous Control (Hs99999905_m1, Thermo Fisher Scientific, Waltham, MA, USA) and HDAC1, TaqMan Gene Expression Assays (Hs00606262_g1, Thermo Fisher Scientific, Waltham, MA, USA).

Statistical analysis. All statistical analyses were performed using the SPSS version 23 software (IBM SPSS Statistics; IBM Corporation, Armonk, NY, USA). The associations between maspin expression status and clinicopathological factors, and the correlation between the subcellular localization of maspin and HDAC1 expression status were evaluated by the Chi-square test. We compared the relative HDAC 1 mRNA expression by using a one-way analysis of variance (ANOVA) and the Tukey honest significant difference (HSD) test. For the survival analyses, cancer relapse (local recurrence or distant recurrence) was used to calculate disease-free survival (DFS). DFS was defined as the period from the date of primary surgery to the date of clinical or 

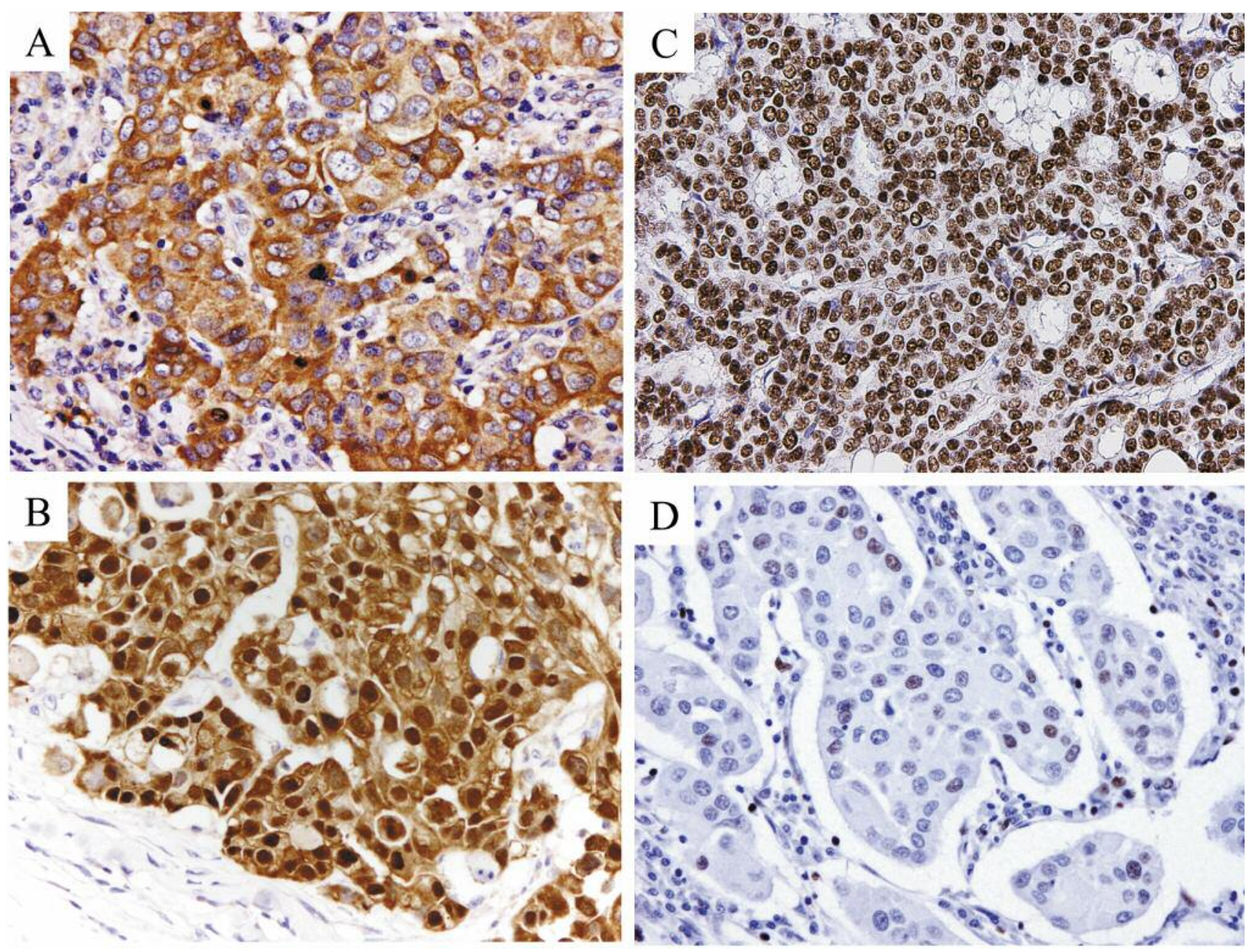

Figure 1. Immunohistchemical staining patterns of maspin in invasive carcinoma of the breast. A: Cytoplasmic-only expression of maspin. B: Pancellular (combined nuclear and cytoplasmic) expression of maspin. Immunohistchemical staining of HDAC1 in invasive carcinoma of the breast. C: High expression of HDAC1 (intensity score: 3, proportion score: 4). D: Low expression of HDAC1 (intensity score: 2, proportion score: 2).

pathological cancer relapse. In the calculation of DFS, patients were censored at the time of their last cancer-free follow-up or at the time of death due to a reason unrelated to cancer. Survival curves were plotted by the Kaplan-Meier method and differences in DFS were tested using the log-rank test. All tests were twosided, and differences were considered significant at $p$-values $<0.05$.

\section{Results}

Clinicopathological characteristics. The median age of the 164 patients at the time of operation was 60 years (range $=32$ 90 years), and the median follow-up time was 68.5 months (range=9-104 months). The positive rates of ER, PgR and HER 2 were $82.3 \%, 64.6 \%$ and $16.5 \%$, respectively. Twentyone patients $(12.8 \%)$ experienced recurrence, and three patients $(1.8 \%)$ deceased of breast cancer progression.
Subcellular localization of maspin. Representative immunohistochemical staining patterns of maspin expression are shown in Figure 1A and B. Subcellular localization of maspin expression was as follows: cytoplasmic-only staining (50 cases, 30.5\%), pancellular (combined nuclear and cytoplasm) staining (23 cases, 14.0\%), and no staining (91 cases, $55.5 \%$ ). Nuclear-only staining was not observed.

Relationship between clinicopathological features and subcellular localization of maspin. The associations between the clinicopathological features and subcellular localization of maspin expression are summarized in Table I. Cytoplasmic-only expression of maspin significantly correlated with higher histological grade $(p=0.004)$ and negative PR status $(p=0.003)$. Pancellular expression of 
Table I. Association between subcellular localization of maspin and clinicopathological characteristics.

\begin{tabular}{|c|c|c|c|c|c|c|c|}
\hline \multirow[t]{2}{*}{ Factors } & \multirow{2}{*}{$\begin{array}{c}\text { Total } \\
(\mathrm{N}=164)\end{array}$} & \multicolumn{3}{|c|}{ Cytoplasmic-only expression of maspin } & \multicolumn{3}{|c|}{ Pancellular expression of maspin } \\
\hline & & $\begin{array}{c}\text { Positive } \\
(\mathrm{N}=50)\end{array}$ & $\begin{array}{l}\text { Negative } \\
(\mathrm{N}=114)\end{array}$ & $p$-Value & $\begin{array}{c}\text { Positive } \\
(\mathrm{N}=23)\end{array}$ & $\begin{array}{l}\text { Negative } \\
(\mathrm{N}=141)\end{array}$ & $p$-Value \\
\hline \multicolumn{8}{|l|}{ Age (years) } \\
\hline$\leq 50$ & 48 & 10 & 38 & 0.084 & 9 & 39 & 0.262 \\
\hline$>50$ & 116 & 40 & 76 & & 14 & 102 & \\
\hline \multicolumn{8}{|c|}{ Pathological T (mm) } \\
\hline$\leq 20$ & 126 & 41 & 85 & 0.299 & 23 & 103 & 0.005 \\
\hline$>20$ & 38 & 9 & 29 & & 0 & 38 & \\
\hline \multicolumn{8}{|c|}{ Lymph node metastasis } \\
\hline Absent & 121 & 39 & 82 & 0.416 & 18 & 103 & 0.598 \\
\hline Present & 43 & 11 & 32 & & 5 & 38 & \\
\hline \multicolumn{8}{|c|}{ Histological grade } \\
\hline I & 34 & 5 & 29 & 0.004 & 9 & 25 & 0.064 \\
\hline II & 84 & 23 & 61 & & 9 & 75 & \\
\hline III & 46 & 22 & 24 & & 5 & 41 & \\
\hline \multicolumn{8}{|c|}{ Estrogen receptor } \\
\hline Positive & 135 & 39 & 96 & 0.337 & 19 & 116 & 0.968 \\
\hline Negative & 29 & 11 & 18 & & 4 & 25 & \\
\hline \multicolumn{8}{|c|}{ Progesterone receptor } \\
\hline Positive & 106 & 24 & 82 & 0.003 & 16 & 90 & 0.594 \\
\hline Negative & 58 & 26 & 32 & & 7 & 51 & \\
\hline \multicolumn{8}{|l|}{ HER2 } \\
\hline Positive & 27 & 10 & 17 & 0.419 & 3 & 24 & 0.633 \\
\hline Negative & 137 & 40 & 97 & & 20 & 117 & \\
\hline
\end{tabular}

maspin significantly correlated with lower pathological tumor classification $(p=0.005)$.

Survival analysis according to maspin expression status. For the survival analysis, we classified the subcellular localization of maspin expression into three groups. DFS curve for the patients is shown in Figure 2. The 5-year DFS rates of the cytoplasmic-only staining group, pancellular staining group and no staining group were $86.5 \%(95 \%$ confidence interval $[\mathrm{CI}]=72.4-93.7 \%), 95.7 \%(95 \% \mathrm{CI}=72.9-$ $99.4 \%)$ and $90.5 \%(95 \% \mathrm{CI}=81.8-95.1 \%)$, respectively. According to the log-rank test, the cytoplasmic-only staining group showed significantly shorter DFS compared to the pancellular staining group $(p=0.043)$.

Immunohistochemical findings of HDAC1. HDAC1 was observed in the nucleus and was expressed in both tumor cells and normal epithelium (Figure 1C and D). Regarding the staining intensity of $\mathrm{HDAC} 1$, the proportion of strong, moderate and weak staining was $35.4 \%, 43.3 \%$ and $18.9 \%$, respectively. The proportion of HDAC1-positive cells was as follows: $58.5 \%$ (96 cases) at $\geq 66 \%, 21.3 \%$ (35 cases) at $33-66 \%, 12.8 \%$ (21 cases) at $10-33 \%$, and $4.9 \%$ ( 8 cases) at $<10 \%$. Four $(2.4 \%)$ cases showed no expression of HDAC1. Thus, high expression

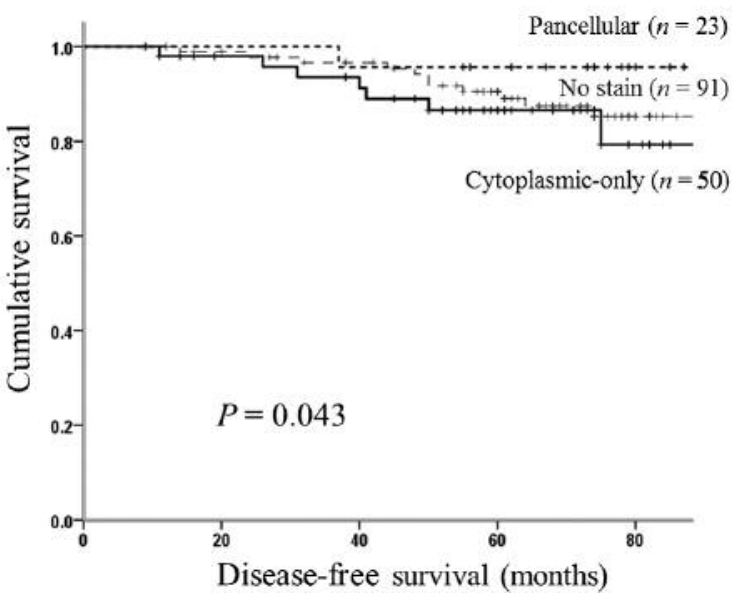

Figure 2. Kaplan-Meier survival curve for disease-free survival of the 164 patients according to subcellular localization of maspin expression (cytoplasmic-only, pancellular, and no staining groups).

of HDAC1 was observed in 98 cases (59.8\%) and it was correlated only with lower histological grade $(p=0.027)$. There was no significant difference in DFS between the patients showing high and low expression of HDAC1. 
A
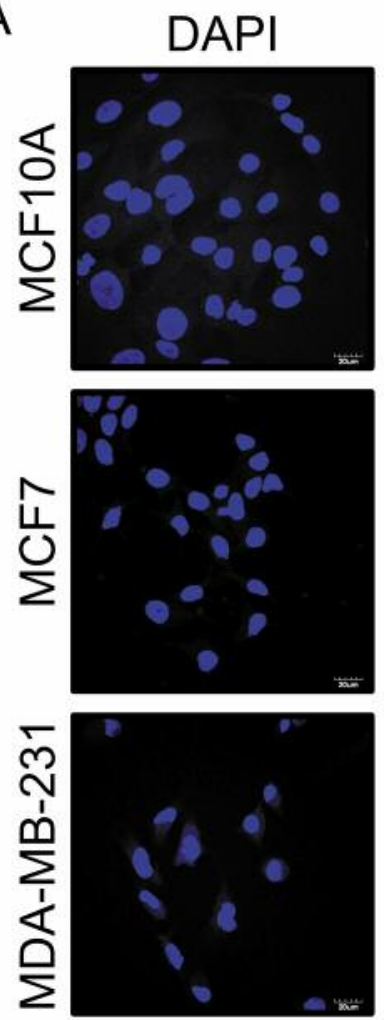

Maspin
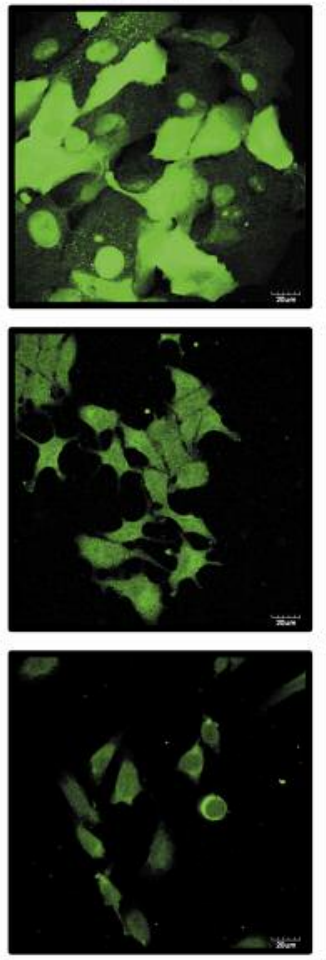

Merge
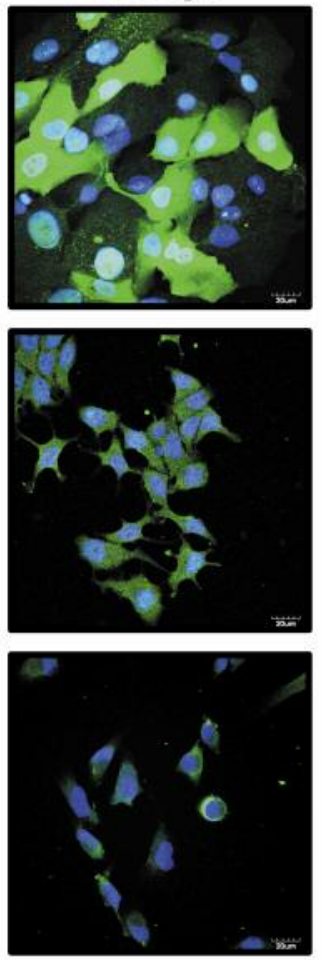

B

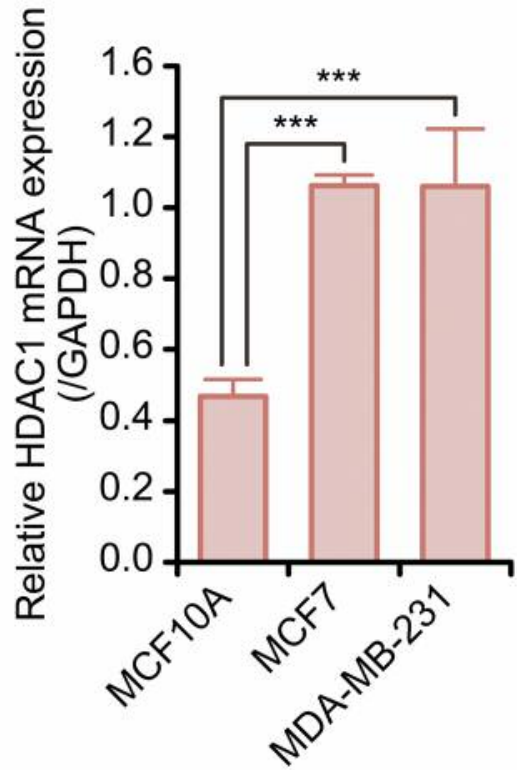

Figure 3. Association between HDAC1 mRNA expression and subcellular localization of maspin in normal breast epithelial cell line and breast cancer cell lines. A: Subcellular localization of maspin in MCF10A, MCF7, and MDA-MB-231 cells was analyzed by immunofluorescence studies with maspin-specific antibody. Maspin (Alexa 488, green) and nuclei (DAPI, blue) signals were detected by laser confocal microscopy. B: Expression of HDAC1 mRNA in MCF10A, MCF7 and MDA-MB-231 cells was examined by qPCR. HDAC1 mRNA expression level was normalized to the expression of GAPDH. Data are shown as mean \pm standard deviation $(S D)(n=3) . * * * p<0.001$ by one-way ANOVA and Tukey's HSD test.

Association between subcellular localization of maspin and HDACl expression. The correlation between subcellular localization of maspin and HDAC1 expression is summarized in Table II. Cytoplasmic-only subgroup was significantly positively correlated with high expression of HDAC1 compared to the pancellular subgroup $(p<0.001)$ and compared to the no staining subgroups $(p=0.004)$. The pancellular subgroup showed a significant positive correlation with the low expression of HDAC1 compared to the no staining subgroup $(p=0.028)$.

Association between subcellular localization of maspin and HDAC1 mRNA expression in breast cancer cells. Immunofluorescence analysis showed that maspin protein was localized mainly in the cytoplasm in MCF7 and MDAMB-231 cells, whereas it was localized in both the nucleus and cytoplasm in MCF10A cells (Figure 3A). The protein levels of maspin in MCF7 and MDA-MB-231 cells were lower than those in MCF10A cells (data not shown). The
Table II. Correlation between subcellular localization of maspin and HDAC1 expression.

\begin{tabular}{lccc}
\hline & \multicolumn{2}{c}{ HDAC1 expression } & \\
\cline { 2 - 3 } & High & Low & \\
& & & \\
& -Value \\
\hline Maspin expression & & 10 & $<0.001$ \\
\hline Cytoplasmic-only & 40 & 16 & \\
Pancellular & 7 & 10 & 0.004 \\
\hline Cytoplasmic-only & 40 & 40 & \\
No staining & 51 & 16 & 0.028 \\
\hline Pancellular & 7 & 40 & \\
No staining & 51 & & \\
\hline
\end{tabular}

HDAC1: Histone deacetylase 1.

HDAC1 mRNA levels were significantly up-regulated in MCF7 and MDA-MB-231 cells compared to those in MCF10A cells $(p<0.001)$ (Figure 3B). 


\section{Discussion}

We previously reported that cytoplasmic-only expression of maspin was an independent poor prognostic indicator in breast cancer patients (6) and that its expression was down-regulated through the progression from ductal carcinoma in situ to invasive ductal carcinoma using immunohistochemical analysis (7). In the largest series of breast cancer patients by Mohsin et $a l$., it has been reported that cytoplasmic staining of maspin was related to ER and PgR negativity, high S-phase fraction and aneuploidy, and that the nuclear staining of maspin was associated with good prognostic factors (4). The same study also described that cytoplasmic staining showed some nuclear staining in all but nine of the cases, and therefore a very few cases of cytoplasmic-only staining were observed (4). This discrepancy of subcellular localization of maspin may be attributable to the difference of the antibody used. In the present study, cytoplasmic-only expression of maspin was observed in $30.5 \%$ of the 164 samples, which is a frequency similar to our previous investigation's finding (27.4\%) (6). Our present study also confirmed that cytoplasmic-only expression of maspin was significantly correlated with shorter DFS despite the relatively short median follow-up period, higher histological grade and PgR negativity, as we observed in the previous investigation (6). In addition, we revealed for the first time that pancellular expression of maspin was not correlated with DFS or a marker for an aggressive phenotype (i.e., with a higher histological grade, larger tumor size, ER/PgR negativity, and lymph node metastasis). Goulet et al. reported that the nuclear localization of maspin was required for its tumor and metastasis suppressor function and that tumor cells expressing nucleus-excluded, cytoplasmic-only maspin were more metastatic than the controls in an in vivo model system (10). They speculated that cytoplasmic-only - not pancellular expression of maspin might be correlated with an aggressive phenotype, which supports our present and previous findings using clinical samples. As the tumor suppressor activity of maspin requires its nuclear localization, it is important to elucidate how maspin gets recruited to chromatin and regulates gene expression. HDAC1, a member of the class I HDACs, is the most extensively characterized protein of this family. An increased deacetylation of histones leads to an increase of cell proliferation and migration, angiogenesis and invasion by reducing the transcription of tumor suppressor genes (17). Maspin is the only endogenous polypeptide HDAC1 inhibitor identified thus far and the endogenous inhibition of HDAC1 has been considered one of the tumor-suppressive mechanisms of maspin (11). We therefore speculated that cases with cytoplasmic-only expression of maspin would frequently show high expression of HDAC1.

To our knowledge, the present findings demonstrated for the first time that cytoplasmic-only expression of maspin positively correlated with high HDAC1 expression, and that pancellular expression of maspin is positively associated with low HDAC1 expression compared to no expression in clinical samples. Our results also demonstrated that two human breast cancer cell lines with cytoplasmic-predominant expression of maspin showed higher HDAC1 expression compared to human mammary epithelial cell lines with pancellular expression of maspin. This observation in the in vitro experiment supports our data from the clinical samples, suggesting that subcellular localization of maspin could play an important role in the regulation of HDAC1. However, the molecular interaction between maspin and HDAC1 may not be mutually exclusive. In fact, $\mathrm{HDAC} 1$ target genes include $\mathrm{p} 27^{\mathrm{KIP} 1}, \mathrm{p} 21^{\mathrm{WAF} / \mathrm{CIP} 1}$ and Bax, whereas maspin interact with glutathione S-transferase (GST), interferon-responsive factor 6 (IRF6) and pro-urokinase type plasminogen activator (prouPA) in an HDAC1-independent manner (2). Further investigation is necessary to clarify the precise mechanism and to determine the significance of cytoplasmic-only expression of maspin.

In conclusion, our present results demonstrated that cytoplasmic-only (not pancelluar expression) expression of maspin was an unfavorable prognostic indicator and was positively correlated with high HDAC1 expression in human breast cancer tissue samples as well as human breast cancer cell lines. These results support the hypothesis that the nuclear localization of maspin in cancer cells is necessary for its inhibition of tumor progression and that an endogenous inhibition of HDAC 1 could be one of the tumorsuppressive mechanisms of maspin.

\section{Conflicts of Interest}

The Authors confirm that there are no conflicts of interest.

\section{Acknowledgements}

The Authors are grateful to Kazuo Yashima and Kazuko Fukushima for their excellent technical assistance with the processing of the pathological specimens. This work was supported by JPSS KAKENHI GRANT Number JP16K10458.

\section{References}

1 Zou Z, Anisowicz A, Hendrix MJ, Thor A, Neveu M, Sheng S, Rafidi K, Seftor E and Sager R: Maspin, a serpin with tumorsuppressant activity in human mammary epithelial cells. Science 263: 526-529, 1994.

2 Bodenstine TM, Seftor RE, Khalkhali-Ellis Z, Seftor EA, Pemberton PA and Hendrix MJ: Maspin: molecular mechanisms and therapeutic implications. Cancer Metastasis Rev 31: 529$551,2012$.

3 Maass N, Teffner M, Rösel F, Pawaresch R, Jonat W, Nagasaki $\mathrm{K}$ and Rudolph P: Decline in the expression of the serine proteinase inhibitor maspin is associated with tumour progression in ductal carcinomas of the breast. J Pathol 195: 321-326, 2001. 
4 Mohsin SK, Zhang M, Clark GM and Craig Allred D: Maspin expression in invasive breast cancer: association with other prognostic factors. J Pathol 199: 432-435, 2003.

5 Kim DH, Yoon DS, Dooley WC, Nam ES, Ryu JW, Jung KC, Park HR, Sohn JH, Shin HS and Park YE: Association of maspin expression with the high histological grade and lymphocyte-rich stroma in early-stage breast cancer. Histopathology 42: 37-42, 2003.

6 Lee MJ, Suh CH and Li ZH: Clinicopathological significance of maspin expression in breast cancer. J Korean Med Sci 21: 309314, 2006.

7 Umekita Y, Ohi Y, Sagara Y and Yoshida H: Expression of maspin predicts poor prognosis in breast cancer patients. Int J Cancer 100: 452-455, 2002.

8 Umekita Y and Yoshida H: Expression of maspin is up-regulated during the progression of mammary ductal carcinoma. Histopathology 42: 541-545, 2003.

9 Umekita Y, Ohi Y, Souda M, Rai Y, Sagara Y, Sagara Y, Tamada $\mathrm{S}$ and Tanimoto A: Maspin expression is frequent and correlates with basal markers in triple-negative breast cancer. Diagn Pathol 6: $36,2011$.

10 Goulet B, Chan G, Chambers AF and Lewis JD: An emerging role for the nuclear localization of maspin in the suppression of tumor progression and metastasis. Biochem Cell Biol 90: 22-38, 2012.

$11 \mathrm{Li} \mathrm{X}$, Yin S, Meng Y, Sakr W and Sheng S: Endogenous inhibition of histone deacetylase 1 by tumor-suppressive maspin Cancer Res 66: 9323-9329, 2006.
12 Kaplun A, Dzinic S, Bernardo $M$ and Sheng S: Tumor suppressor maspin as a rheostat in HDAC regulation to achieve the fine-tuning of epithelial homeostasis. Crit Rev Eukaryot Gene Expr 22: 249-258, 2012.

13 Lakhani SR, Ellis IO, Schnitt SJ, Tan PH and van de Vijver MJ: WHO Classification of Tumours of the Breast, Fourth Edition. IARC, Lyon, 2012.

14 Elston CW and Ellis IO: Pathological prognostic factors in breast cancer. I. The value of histological grade in breast cancer: experience from a large study with long-term follow-up. Histopathology 19: 403-410, 1991.

15 Sobin LH, Gospodarowicz MK and Wittekind C: TNM Classification of Malignant Tumours, Seventh Edition, UICC, Blackwell Publishing Ltd, 2010.

16 Seo J, Min SK, Park HR, Kim DH, Kwon MJ, Kim LS and Ju YS: Expression of histone deacetylases HDAC1, HDAC2, HDAC 3 , and HDAC6 in invasive ductal carcinomas of the breast. J Breast Cancer 17: 323-331, 2014.

17 Glozak MA and Seto E: Histone deacetylases and cancer. Oncogene 26: 5420-5432, 2007.

Received June 28, 2017

Revised July 5, 2017

Accepted July 6, 2017 